\title{
Studying BDNF/TrkB signaling: High-throughput microfluidic gene expression analysis from rare or limited samples of adult and aged central neurons
}

\author{
Arup R Nath ${ }^{1}$, Roy Drissen ${ }^{2}$, Fei Guo ${ }^{1}$, Claus Nerlov ${ }^{2}$ and Liliana Minichiello", * \\ 1Department of Pharmacology, University of Oxford, Oxford OX1 3QT, UK. \\ ${ }^{2}$ MRC Molecular Haematology Unit, Weatherall Institute of Molecular Medicine, University of \\ Oxford, Oxford OX3 9DS, UK.
}

\section{* Corresponding author}

Dr. Liliana Minichiello

Reader in Cellular and Molecular Neuroscience

Department of Pharmacology

University of Oxford

Mansfield Road

Oxford, OX1 3QT

United Kingdom

Email: liliana.minichiello@pharm.ox.ac.uk

Phone: 441865271884

Running title: Microfluidic gene expression analysis from limited number of adult and aged central neurons 


\begin{abstract}
High-throughput next generation sequencing technologies are an invaluable tool to gain insight into the transcriptional states of large cohorts of cells. Such data can help to shed light on the organisation of tissues and pathways under normal and pathological conditions. In our case, we are using the above technology to decipher how the enkephalinergic medium spiny neurons (MSNs) of the striatum adapt to aging in the presence or absence of BDNF/TrkB signalling. However, sequencing data must be validated, ideally with an alternative method that interrogates the transcriptional state of cells, and is able to detect gene expression in rare single cells or bulk cells with high sensitivity. Thus, we have optimized a protocol for high-throughput microfluidic analysis (Fluidigm Dynamic Array Integrated Microfluidic Circuit (IFC)) to validate RNA sequencing data from limited number of adult and aged sorted neurons. We here describe this protocol in detail.
\end{abstract}

\title{
Keywords
}

High-throughput; microfluidic; Fluidigm; TaqMan; bulk cell; gene expression; validation; RNA sequencing, RT-PCR, quantitative, heatmap. 


\section{Introduction}

Analysing and understanding gene expression variability in neurons is central to understanding neuronal development and heterogeneity, and how neurons and brain structures adapt to myriad stimuli-including pathological conditions [1]. However, a major limitation to this analysis is the difficulty in isolating live aged neurons such that analyses can be performed. The bulk-cell neuronal RNA sequencing described in the previous chapter allows us to determine the transcriptional state of specific neurons, namely enkephalinergic neurons of the striatum that depend on neurotrophin signalling for their function [2]. This data is able to provide a large amount of information regarding the unique molecular make up of these neurons, particularly how gene expression changes in response to aging. The changes in gene expression provide clues as to how aging is affecting these neurons and how they have in turn adapted to this stressor [2]. This data, however, needs to be validated before any inferences can be made regarding in vivo states.

The gold-standard for gene expression analysis remains real-time quantitative reverse transcription polymerase chain reaction (RT-PCR) [3]. Here we describe the use of the TaqMan assay system in conjunction with microfluidic technology (Fluidigm) to simultaneously perform RT-PCR on up to 192 unique bulk-cell samples for up to 96 unique genes. Performing the microfluidic gene expression analysis can confirm, with a high degree of certainty, whether the genes of interest are indeed regulated in response to the stimulus of interest, thus validating the RNA sequencing data. Although we have applied it here as a means of validation, this analysis is robust enough to be performed as a stand-alone method to assay gene expression changes across cells, tissues, and disease states.

The TaqMan Assay system relies on the use of fluorogenic probes paired with conventional forward and reverse primers. The probes contain at their 5' end a 6-carboxyfluorescein (FAM) 
fluorescent probe and a non-fluorescent quencher at their 3' end along with a minor groove binding moiety [3] [4]. TaqMan chemistry relies on the 5' exonuclease activity of the Taq polymerase. Following denaturation and annealing of primers and probes, Taq-mediated extension begins. When Taq encounters the probe the exonuclease activity results in the permanent separation of the FAM fluorescent molecule from the 3' quencher [4] [5]. This in turn causes the fluorescent signal to increase proportionally to the number of amplicons generated. The Fluidigm Biomark system allows for the accurate detection of this fluorescence in the microfluidic chip. The fluorescence increase is used to determine the Ct-values, which can then be normalised to reference genes to allow for further statistical analyses [6] [7]. Selecting the appropriate reference genes for normalisation will require the experimenter to screen a panel of genes to determine which one is the most reliable under the specific experimental conditions chosen.

This chapter will describe how we process bulk cells obtained from acute striatal neuron suspensions using fluorescence activated sorting for microfluidic gene expression analysis. We then describe how to process the sample in preparation for loading onto the microfluidic circuit. This is then followed by examples of how the high-throughput RT-PCR data can be processed, analysed, and presented. 


\section{MATERIALS}

\section{REAGENTS}

- Hibernate A low fluorescence (BrainBits, HA-Lf)

- $\quad$ CellsDirect ${ }^{\text {TM }}$ One-Step qRT-PCR Kit (Thermo Fisher, 11753100), that includes:

- CellsDirect 2x Reaction Mix

- Ambion's SUPERase 12-In

- RT/Taq (SS III Platinum) Mix

- 20X TaqMan® Gene Expression Assay (Life Technologies)

- 2X Assay Loading Reagent (Fluidigm PN 100-7611)

- $\quad$ TaqMan Universal PCR Master Mix (2X) (Life Technologies PN 4304437)

- 20X GE Sample Loading Reagent (Fluidigm PN 100-7610)

- RNaseZap (Ambion, AM9780)

- DNA-OFF (Takara Bio, 9036)

- Nuclease-free water (Ambion, AM9930)

- 8-strip microcentrifuge tube, $0.2 \mathrm{~mL}$ (Starlab I1402-2908)

- $\quad$ Filter tips (Axygen, $10 \mu \mathrm{L}$, TF-300-L-R-S; $20 \mu \mathrm{L}$, TF-20-L-R-S; $200 \mu \mathrm{L}$, TF-200-L-R-S; $1,000 \mu L, T F-1000-L-R-S)$ 


\section{EQUIPMENT}

- BioMark (Fluidigm)

- $\quad$ IFC Controller MX/RX/HX (Fluidigm) (48.48;192.24; 96.96 respectively)

- $\quad$ IFC 48.48; 192.24; 96.96 (Fluidigm) of choice

- DNA Engine Peltier Thermal Cycler (Bio-Rad)

- Clean Room

- Hood

- FACSAria Fusion Cell Sorter

- 8-channel pipettor $(1-10 \mu \mathrm{L})$ 12-channel pipettor $(20-200 \mu \mathrm{L})$

- Refrigerated table-top centrifuge (Eppendorf 5417R)

- $\quad$ Fixed-angle rotor (A-4-44)

- $\quad-80^{\circ} \mathrm{C}$ Freezer

- $-20^{\circ} \mathrm{C}$ freezer

- Fluidigm Software for IFC

- Fluidigm Software for Gene Expression Analysis

- Microsoft Excel

- GENE-E or Morpheus (Broad Institute) 
Tissue dissection/dissociation and preparation of a cell suspension for FACS

1. For this part, please refer to chapter \#1. The only difference is that we will now be collecting 50 cells instead of 200 cells.

Preparation of lysis/preamplification buffer for collecting 50 cells (timing 1 hour)

2. The preparation of the lysis/preamplification buffer must be performed in the clean room. All reagents must be kept as cool as possible to ensure that they do not degrade. If possible, only retrieve required reagents from the $-20^{\circ} \mathrm{C}$ freezer where they are stored immediately before they are required, allowing only the time required to thaw certain reagents. (See Note 1).

3. Preparation of the lysis buffer requires preparation of 0.2x TaqMan Assay Mix. The desired number of assays (determined by the experiment and the number of assay inlets in the Fluidigm chip) are added into TE buffer to create an assay mix that is diluted 100 times. (See Note 2).

4. For a bulk cell experiment where there are 24 genes of interest, a $100 \mu \mathrm{L}$ mix is made by adding $1 \mu \mathrm{L}$ of each TaqMan assay to $76 \mu \mathrm{L}$ of TE buffer to obtain an $0.2 x$ TaqMan Assay Mix. Depending on the number of samples that need to be prepared, you may need to make 200 or $300 \mu \mathrm{L}$ of the assay mix.

5. The total volume of the lysis buffer, per 50-cell sample, will be $10 \mu \mathrm{L}$. The lysis buffer consists of:

\begin{tabular}{|l|l|}
\hline Reagent & Volume \\
\hline CellsDirect 2x Reaction Mix & $5 \mu \mathrm{L}$ \\
\hline Ambion's SUPERase 12-In & $0.1 \mu \mathrm{L}$ \\
\hline $0.2 x$ Taqman Assay Mix & $2.5 \mu \mathrm{L}$ \\
\hline
\end{tabular}




\begin{tabular}{|l|l|}
\hline RT/Taq (SS III Platinum) Mix & $1.2 \mu \mathrm{L}$ \\
\hline TE buffer & $1.2 \mu \mathrm{L}$ \\
\hline
\end{tabular}

6. Scale this up according to the number of samples that will be collected, while also including coverage.

7. Make sure that the lysis buffer is always made fresh. Making it up days in advance may risk some of the reagents and enzymes becoming degraded or deactivated.

8. Dispense $10 \mu \mathrm{L}$ of the lysis buffer into a clearly labelled $0.2 \mathrm{~mL}$ thin-walled PCR tube for each 50-cell sample. (See Note 3).

9. We use a $100 \mu \mathrm{m}$ nozzle on a FACSAria Fusion Cell Sorter so each cell will be sorted in $0.0035 \mu \mathrm{L}$ volume. Thus 50 cells will make up approximately $0.175 \mu \mathrm{L}$ of volume.

10. Once the lysis buffer is made it needs to remain on ice.

11. In preparation for assay loading on the Fluidigm IFC chip (after preamplifcation), aliquot 3 $\mu \mathrm{L}$ of each TaqMan assay into clearly labelled $0.2 \mathrm{~mL}$ thin-walled PCR tubes, and store them at $-20^{\circ} \mathrm{C}$.

\section{FACS (timing 1-2 hours)}

12. Collect 50 cells into the early prepared $0.2 \mathrm{~mL}$ thin-walled PCR tube (from step 8 ). For the gating and sample processing please refer to Chapter\#1.

\section{cDNA synthesis and pre-amplification (timing 2-3 hours)}

13. cDNA synthesis and pre-amplification is performed simultaneously using the following steps:

\begin{tabular}{|c|l|l|l|}
\hline STEP & Description & TEMPERATURE & DURATION \\
\hline
\end{tabular}




\begin{tabular}{|c|c|c|c|}
\hline 1 & $\begin{array}{c}\text { Reverse } \\
\text { transcription }\end{array}$ & $50^{\circ} \mathrm{C}$ & $15 \mathrm{~min}$ \\
\hline 2 & $\begin{array}{c}\text { Inactivation of } \\
\text { RTase and } \\
\text { activation of Taq }\end{array}$ & $95^{\circ} \mathrm{C}$ & $2 \mathrm{~min}$ \\
\hline 3 & Amplification & $95^{\circ} \mathrm{C}$ & $15 \mathrm{sec}$ \\
\hline 4 & Amplification & $60^{\circ} \mathrm{C}$ & $-\cdots$ min \\
\hline 5 & $\begin{array}{c}\text { repeat steps 3-4 } \\
\text { for 17 more times } \\
(18 \text { total cycles) } \\
\text { Final elongation }\end{array}$ & ----------- \\
\hline 7 & Hold & $4^{\circ} \mathrm{C}$ & 10 min \\
\hline
\end{tabular}

14. The number of pre-amplification cycles must be chosen carefully so as to be within the dynamic range, ie. ensuring that a sufficient number of cycles are selected so that enough cDNA is synthesised but not too many cycles so that too much cDNA is synthesised (which would obscure differences in gene expression levels due to saturation). (See Note 4).

15. Once the process is completed store the tubes at $-20^{\circ} \mathrm{C}$ in preparation for Fluidigm gene expression analysis on the IFC.

\section{cDNA dilution}

16. Thaw the cDNA on ice, and add $40 \mu \mathrm{L}$ TE buffer to each tube. The total volume in each tube should now be $50 \mu \mathrm{L}$. (See Note 5).

\section{Preparation of samples and assays for chip loading (timing 1-2 hours)}

17. Preparation of the cDNA samples and TaqMan assays (the latter were aliquoted in the clean room when the lysis buffer was prepared, see step 11 above) should be exactly as stated in the Fluidigm protocol. (See Note 6). The protocols can be found on the Fluidigm website: https://www.fluidigm.com/documents. 
18. Preparation will differ depending on the type of chip being used.

\section{$\underline{\text { Chip loading and Biomark protocol (2-3 hours) }}$}

19. Load the chip (e.g. 48.48, Figure 1) according to an experimental plan that is appropriate for the analysis that you want to perform. (See Note 7).

20. Insert the loaded chip into Biomark machine and choose the appropriate gene expression analysis (GE) protocol. (See Note 8).

21. Ensure that "auto-exposure" is selected.

22. Run the experiment.

\section{Biomark data handling}

23. The data from the experiment will automatically be saved (Figure 2).

24. Open the "Real-Time PCR Analysis" software to carefully assign assay names and identities to the samples. (See Note 9).

25. Export the dataset ( $\Delta$ Ct values) as a .CSV file.

\section{Gene expression analysis}

26. Convert the .CSV file of the dataset into an .xIsx file for ease of use in Microsoft Excel.

27. The data can be handled in any number of ways depending on what the experiment demands. Below we describe the most common way to represent and evaluate highthroughput gene expression data sets, which is the heat map. However, the processed $\Delta C t$ values (as shown below) can be used to perform statistical analysis between bulk cell groups.

28. To perform the subsequent analysis the $\Delta C t$ values of each sample needs to be clearly separated. Use the "filter" function to filter the dataset for one of the genes-this will now show the $\Delta C t$ values of all the samples for that particular gene. Copy and paste this into a 
new sheet. Repeat this for all the genes. Thus, you should have each sample's expression level ( $\Delta C t$ values) of each gene organized in adjacent columns.

29. Replace each "999" value (which indicates that no expression was detected) with an " $x$ " for analysis purposes. This must be changed to an " $x$ " (or to a high Ct value) or the subsequent analysis will be rendered meaningless.

30. The first quality control step will remove all samples that do not express the reference gene(s). (See Note 10).

31. The expression level of each gene must now be normalised. This is done by normalising the expression of each gene (in each sample) to that sample's expression of a reference gene. As such, subtract each gene's expression level from the expression level of the reference gene:

$=\Delta C t$ (reference gene) $-\Delta C t$ (gene being tested)

32. Now replace all the error "\#VALUE!" cells with " $x$ ". (This will result from samples where the expression of a gene is not detected.)

33. For each gene, calculate the average expression level across all the samples.

34. Now we will centre the expression of each gene around 0 . This allows us to determine gene expression relative to other samples of interest. This is done by subtracting the mean expression of each gene (across all samples) from the expression level of the gene in each sample:

$$
=\Delta C t(\text { gene })-\text { mean }(\Delta C t(g e n e))
$$

35. Now replace the error "\#VALUE!" cells with an empty cell. The empty cell will represent "undetected expression".

36. Import this file into a program that allows for the graphical visualization of the gene expression data. The online open-source software Morpheus (Broad Institute) can be used to generate heat maps and also perform clustering. 


\section{Example of analysis}

37. Expression analysis of Canx and Gusb (reference genes) (Figure 3). 


\section{Acknowledgements}

We would like to thank members of the Minichiello and the Nerlov laboratories for the support they provided. ARN, was supported by a Clarendon Scholarship. FG, was supported by a China Scholarship Council (CSC). This work was supported by a BBSRC grant (BB/L021382/1) to LM.

\section{Competing financial interests}

The authors declare no competing financial interests. 


\section{NOTES}

Note 1: The preparation of the lysis buffer and TaqMan assay mix must be performed in a clean room (in a DNA-free hood) to ensure that there is no contamination. Any extraneous RNA or DNA will confound the final results.

Note 2: The TaqMan assays arrive as at 20x concentration. However, for the protocol described here, the TaqMan assays need to be diluted 100 times (or, to $0.2 x$ ) before it can be used.

Note 3: Be sure to only use hydrophobic markers to label tubes, as ink from other water-soluble markers will dissolve when placed in dry ice and when placed in the Thermal Cycler.

Note 4: From our experience, we find that neurons require more cycles of preamplification than would be required for other cell types. Determining the optimal number of cycles for your cells of interest may require several trials with known housekeeping genes.

Note 5: The diluted (or preamplified) cDNA can be stored at $-20^{\circ} \mathrm{C}$ and can be reused for many future experimental runs.

Note 6: The Fluidigm protocol states that the preparation should be performed in a DNA-free hood, however in our experience we have found that a thoroughly cleaned bench is sufficient.

Note 7: The pipetting must be performed as carefully as possible. Ensure that the samples and assays are pipetted directly to the bottom of each well. It is absolutely crucial that there are no bubbles in any of the wells, as this may make that sample/assay unusable. 
Note 8: The gene expression analysis (GE) protocol will differ according the IFC being used. Take care to ensure that you have selected the proper protocol.

Note 9: This is critical to any further analysis, if the samples or assays are incorrectly labelled the analysis will lead to erroneous conclusions.

Note 10: For bulk cell gene expression, this will be rare. It becomes more important when performing single cell gene expression analysis. 


\section{References}

1. Poulin JF, Tasic B, Hjerling-Leffler J, Trimarchi JM and Awatramani R (2016) Disentangling neural cell diversity using single-cell transcriptomics. Nat Neurosci 19:113141. doi: $10.1038 / \mathrm{nn} .4366$

2. Besusso D, Geibel M, Kramer D, Schneider T, Pendolino V, Picconi B, Calabresi P, Bannerman DM and Minichiello L (2013) BDNF-TrkB signaling in striatopallidal neurons controls inhibition of locomotor behavior. Nat Commun 4:2031. doi: 10.1038/ncomms3031

3. Heid CA, Stevens J, Livak KJ and Williams PM (1996) Real time quantitative PCR. Genome Res 6:986-94.

4. Osman F, Leutenegger C, Golino D and Rowhani A (2007) Real-time RT-PCR (TaqMan) assays for the detection of Grapevine Leafroll associated viruses 1-5 and 9. J Virol Methods 141:22-9. doi: 10.1016/j.jviromet.2006.11.035

5. Holland PM, Abramson RD, Watson R and Gelfand DH (1991) Detection of specific polymerase chain reaction product by utilizing the 5'----3' exonuclease activity of Thermus aquaticus DNA polymerase. Proc Natl Acad Sci U S A 88:7276-80.

6. Livak KJ and Schmittgen TD (2001) Analysis of relative gene expression data using realtime quantitative PCR and the 2(-Delta Delta C(T)) Method. Methods 25:402-8. doi: 10.1006/meth.2001.1262

7. Drissen R, Buza-Vidas N, Woll P, Thongjuea S, Gambardella A, Giustacchini A, Mancini E, Zriwil A, Lutteropp M, Grover A, Mead A, Sitnicka E, Jacobsen SE and Nerlov C (2016) Distinct myeloid progenitor-differentiation pathways identified through single-cell RNA sequencing. Nat Immunol 17:666-76. doi: 10.1038/ni.3412 


\section{Figure Legends}

Figure 1. Typical 48.48 Fluidgm IFC microfluidic chip. Indicated is where the TaqMan gene assays are loaded, 'Assay inlets'. Whereas, the bulk-cell samples are loaded in the 'Sample inlets'. The fluorescence-producing qPCR reactions are performed in the 'Reaction centre'. The proprietary control line fluid is injected into accumulators in the indicated locations prior to chip priming.

Figure 2. Amplification plots generated by the BioMark Real Time PCR analysis software for a completed 48.48 IFC chip run. The Y-axis indicates the change in fluorescence (generated by the FAM-MGB probes), while the X-axis indicates the number of PCR cycles required to reach a given level of fluorescence change. A) Representative amplification of all assayed genes for 3 months old (3M) bulk cell sample. The picture shows good amplification of the genes of interest indicated by the fact that they require between $15-25$ cycles to reach the log growth phase of amplification. B) Same as in (A) but for 8 months old $(8 \mathrm{M})$ bulk cell sample. Note the green and red traces at the bottom right of both graphs $(A, B)$ indicating background/negative samples.

Figure 3. Gene expression analysis of two reference genes at 3 and 8 months of age. (A) $\Delta C t$ values of two reference genes, calcineurin (CANX) and glucuronidase beta (GUSB), from 3 and 8 months old $(3 M, 8 M)$ mice $(n=2$ each age). Shown are three technical replicates for each gene. The raw $\Delta C t$ values were taken directly from the BioMark .csv file and graphed here. (B) The $\Delta C t$ values from $(\mathrm{A})$ were used to generate a heatmap using Morpheus/GENE-E (Broad Institute). 


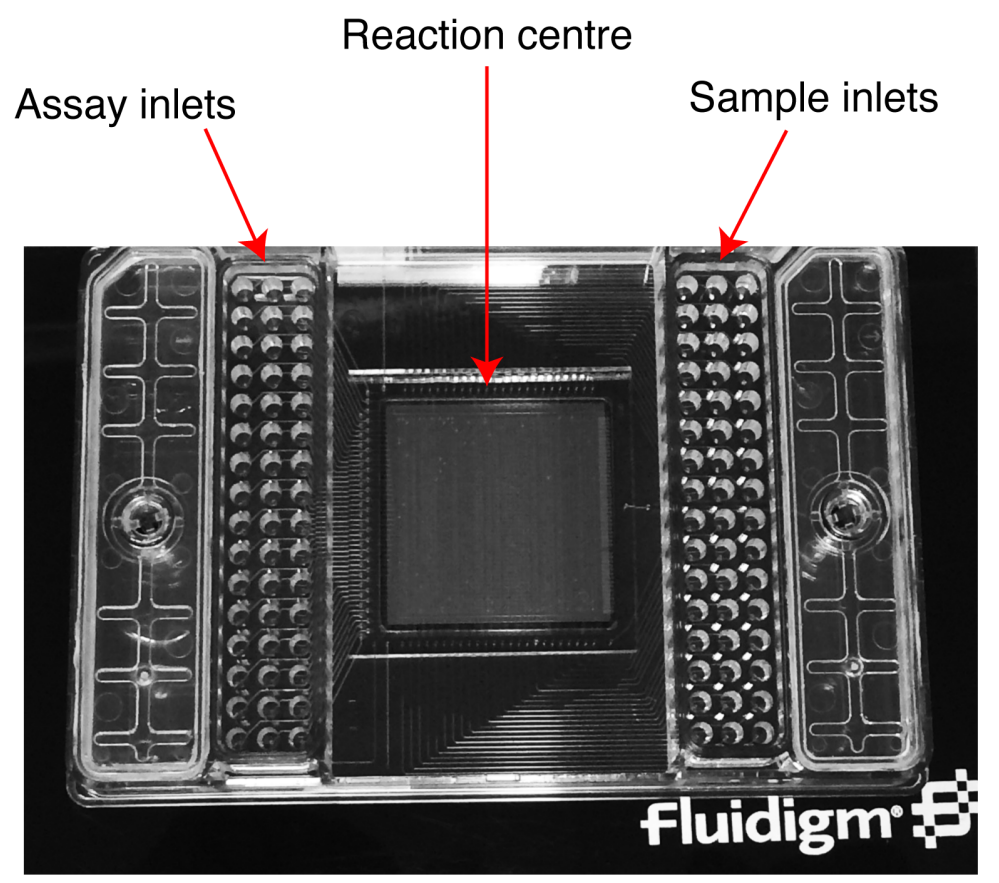

Figure 1 

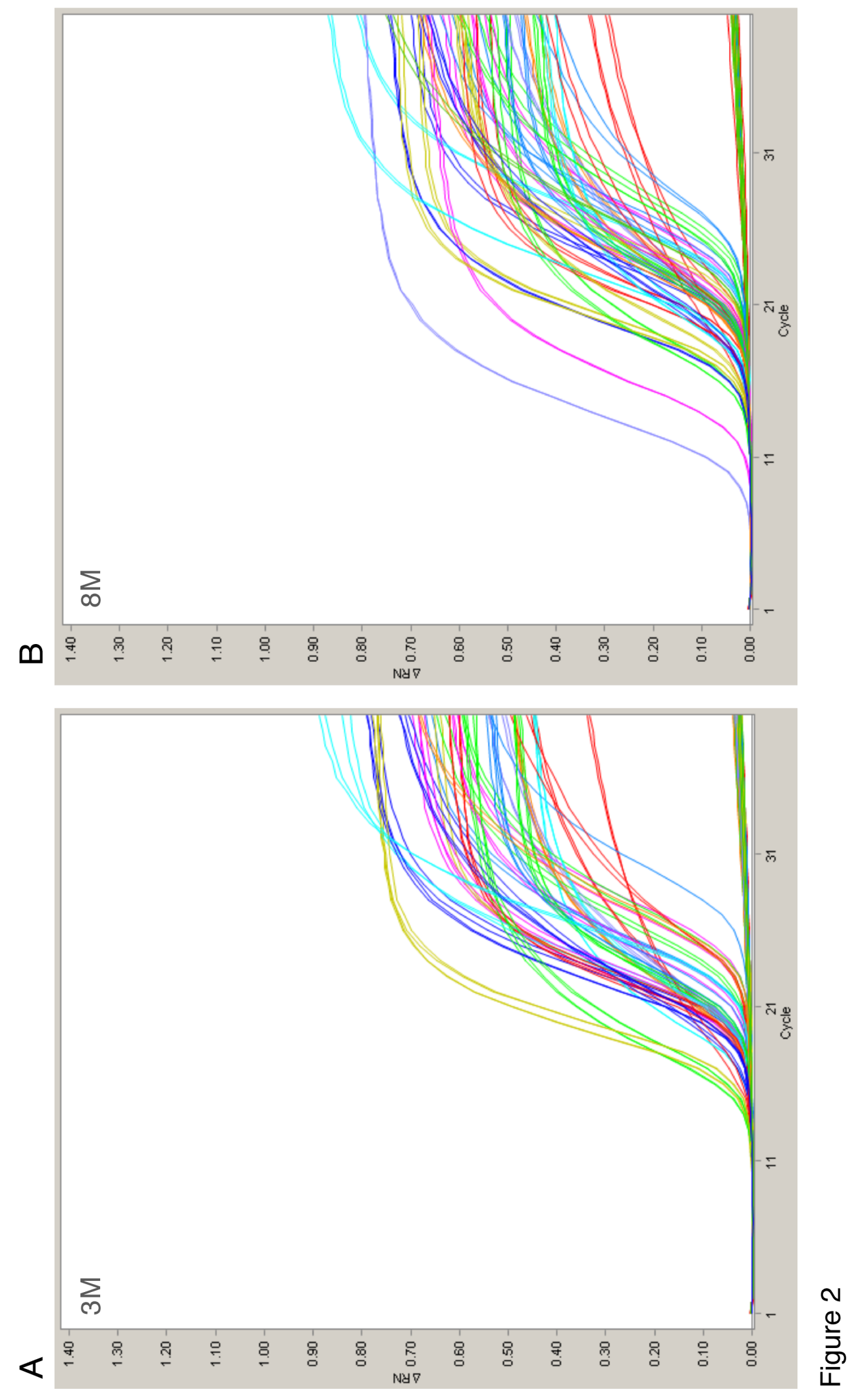


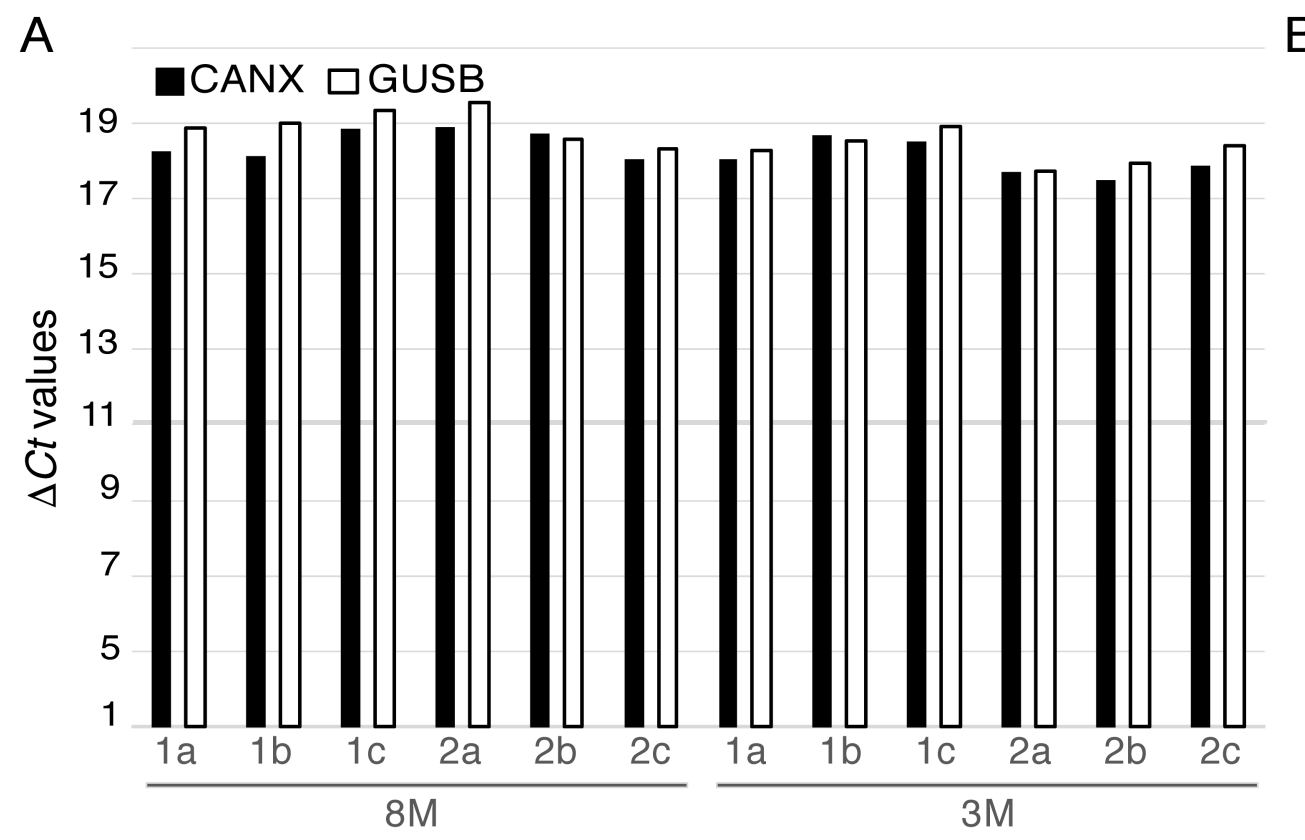

B

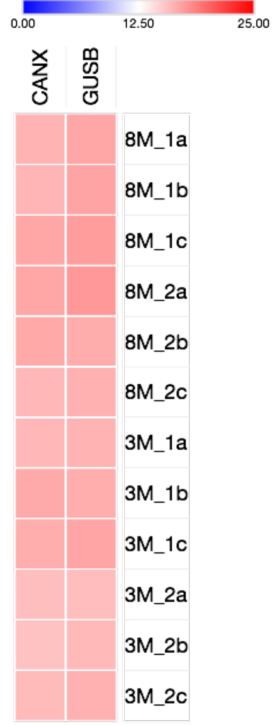

Figure 3 